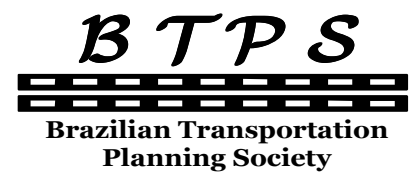

Journal of Transport Literature

Vol. 8, n. 1, pp. 95-108, Jan. 2014

Research Directory

\title{
Modelo teórico de tarifa portuária baseado na contabilidade de custos e gerencial e nas finanças corporativas
}

[Theoretical model for port tariff based on cost and managerial accounting and corporate finance]

\author{
Carlos Henrique Rocha*, Francisco Giusepe Donato Martins, \\ Francisco Gildemir Ferreira da Silva \\ Universidade de Brasília - Brasil, Tribunal de Contas da União - Brasil, \\ Agência Nacional de Transportes Terrestres - ANTT - Brasil
}

Submitted 15 Mar 2012; received in revised form 4 Oct 2012; accepted 27 Dec 2012

\begin{abstract}
Resumo
As tarifas portuárias brasileiras são ainda hoje construídas de forma ad hoc. Sem um modelo teórico de tarifação cede-se lugar, inclusive, para abusos econômicos de exercício de poder de mercado e dificulta o papel das autoridades, especialmente, da Agência Nacional de Transportes Aquaviários (ANTAQ) para atuar sobre os portos organizados, particularmente na promoção de estudos aplicados às definições de tarifas e preços, em confronto com os custos e os benefícios econômicos transferidos aos usuários pelos investimentos realizados. Este artigo propõe um modelo teórico padrão de formação de tarifas portuárias. 0 modelo proposto tem fundamentos de contabilidade de custos, contabilidade gerencial e finanças corporativas. 0 modelo é de fácil entendimento e aplicação, desde que os portos possuam sistemas integrados de custeamento, de contabilidade gerencial e de estatística; sistemas do tipo ERP (Enterprise Resource Planning). Com o modelo os custos totais do porto são parcialmente cobertos, inclusive investimentos. 0 modelo é compatível com a obtenção de lucros.
\end{abstract}

Palavras-Chave: modelo teórico de tarifas portuárias, contabilidade de custos e gerencial, finanças corporativas, lucratividade.

\begin{abstract}
The Brazilian portuary tariffs are still built on ad hoc base. As such, the work of the regulatory agency (Agência Nacional de Transportes Aquaviários, ANTAQ) becomes hard and, for instance, it is thorny to carry out a solid investment analysis. This paper suggests a theoretical model for portuary tariff determination. The model is grounded mainly on tenets of cost and management accounting and of corporate finance. We have assumed that the port has ERP (Enterprise Resource Planning) and statistics systems. The proposed model generates income over total costs. Hence, it is in row with the profitable port argument.
\end{abstract}

Key words: theoretical port pricing model, cost and managerial accounting, corporate finance, profitability.

*Email: chrocha@unb.br.

\section{Recommended Citation}

Rocha, C. H., Martins, F. G. D. and DaSilva, F. G. F. (2014) Modelo teórico de tarifa portuária baseado na contabilidade de custos e gerencial e nas finanças corporativas. Journal of Transport Literature, vol. 8, n. 1, pp. 95-108.

- JTL/RELIT is a fully electronic, peer-reviewed, open access, international journal focused on emerging transport markets and published by BPTS - Brazilian Transport Planning Society. Website www.transport-literature.org. ISSN 2238-1031.

This paper is downloadable at www.transport-literature.org/open-access. 


\section{Introdução}

No período entre 1930 e 1937, a política brasileira se espelhou nas idéias difundidas no mundo, adotando o corporativismo nacionalista e a participação direta do Governo nos setores de infraestrutura (ferrovias, navegação, serviços públicos) e de indústrias básicas (petróleo e aço), e a participação indireta através da política fiscal, do controle de câmbio e das quotas de importação (ROSSETTI et al, 1985). Nesse escopo, o Governo brasileiro editou o Decreto n. $^{\circ}$ 24.508/34, a fim de definir os serviços prestados pelas administrações dos portos organizados e de uniformizar as taxas portuárias, quanto a sua espécie, incidência e denominação.

O Decreto $n .^{\circ} 24.508 / 34$ estabeleceu que as vantagens e os serviços de que o comércio e a navegação usufruírem nos portos organizados serão retribuídos com o pagamento de importâncias, cobradas pelas administrações desses portos e calculadas pela aplicação de taxas estabelecidas para cada porto em uma tarifa aprovada mediante portaria ministerial. A tarifa portuária, por sua vez, foi constituída, para todos os portos, por meio de várias tabelas de taxas (utilização do porto, atracação, capatazia, armazenagem interna, externa, especial e em armazéns gerais, transportes, estiva de embarcações, suprimento do aparelhamento, reboques, suprimento de água para embarcações, serviços acessórios e movimentação das mercadorias), discriminadas em gerais e especiais, em função das vantagens e dos serviços definidos naquele normativo e indicava, também, o responsável pelo pagamento das importâncias decorrentes da aplicação das taxas especificadas.

Dessa forma, o Decreto n. ${ }^{\circ} 24.508 / 34$ definiu o modelo tarifário a ser adotado por todos os portos, considerado como parâmetro um grande porto, devidamente aparelhado. No entanto, foi previsto que para todos os portos as tabelas deveriam ser completadas com as taxas especiais necessárias, com quaisquer outras isenções, ou observações peculiares ao porto. Porém, na tarifa de cada porto, só deveriam figurar as tabelas relativas aos serviços e às vantagens, que ali seriam, ou poderiam ser assegurados ou realizados, pela administração daquele porto.

Aragão (2004) nota que até meados dos anos oitenta, os Estados intervinham fortemente na economia, devido a razões de equidade social e a imperativos do próprio sistema econômico, 
tais como a criação de infraestruturas vultosas não lucrativas ou de lucratividade diferida, a necessidade de se evitar a monopolização de setores da economia e o fomento de regiões menos desenvolvidas. Ainda, conforme o autor, a partir dos anos oitenta iniciou-se uma mudança na atuação do Estado com o início da privatização de empresas públicas.

Tem-se observado, a partir da década de 80, um conjunto de transformações no Estado brasileiro, especialmente, no que diz respeito à intervenção no domínio econômico, intensificado com a implantação do Programa Nacional de Desestatização, por meio da Medida Provisória n. ${ }^{\circ}$ 115/90, que se transformou em Lei n. ${ }^{\circ}$ 8.031/90. A partir de 1995, esse processo se caracterizou, principalmente, pela desestatização de serviços públicos.

Nesse sentido, o setor portuário brasileiro sofreu mudanças a partir do final da década de 80 e início da década de 90, com a extinção da Portobrás e com a edição da Lei n. ${ }^{\circ}$ 8.630/93, denominada de lei de modernização dos portos. Esta Lei atribui à União o direito de explorar o porto organizado, diretamente ou mediante concessão sempre precedida de licitação. Assegura, também, a quem interessar o direito de construir, reformar, ampliar, melhorar, arrendar e explorar instalação portuária, mediante: (a) contrato de arrendamento; e (b) autorização, cada qual para casos específicos descritos em Lei. Portanto, incentivou a competição e a parceria público-privado por meio do aumento dos investimentos e de gestão empresarial na operação portuária, de modo a proporcionar ganhos de produtividade e redução de tarifas, consoante Pêgo Filho et al. (1999).

A Lei $n .^{\circ}$ 8.630/93, na essência, promoveu uma reforma estrutural e gerencial na exploração dos portos e das instalações portuárias. No contexto desse processo de mudanças foi prevista a criação do Órgão Gestor de Mão-de-Obra do Trabalhador Portuário Avulso (OGMO), com o intuito de se extinguir o monopólio do mercado dos trabalhadores avulsos e de se racionalizar o uso da mão-de-obra. Além disso, no âmbito do porto organizado foi obrigatoriamente instituída a Administração do Porto e o Conselho de Autoridade Portuária (CAP). Ou seja, com a Lei, e em linha com o modelo internacional da época, os portos deixaram de ser operadores portuários e, grosso modo, ficaram exclusivamente por conta da gestão da atividade portuária1; isso implica que os portos não têm custos fabris.

A administração do porto tem a competência, entre outras coisas, de: (a) fixar os valores e arrecadar a tarifa portuária; e (b) fiscalizar as operações portuárias, zelando para que os

\footnotetext{
${ }^{1}$ Para uma discussão sobre a reforma portuária ver Castro (2000).
} 
serviços se realizem com regularidade, eficiência, segurança e respeito ao meio ambiente. Já ao CAP compete, principalmente: (a) promover a racionalização e a maximização do uso das instalações portuárias; (b) fomentar a ação industrial e comercial do porto; (c) zelar pelo cumprimento das normas de defesa da concorrência; (d) homologar os valores das tarifas portuárias; e (e) estimular a competitividade. Pode-se observar que esses aspectos visam estimular a prática de livre concorrência para aumento da eficiência e produtividade, com a conseqüente redução dos custos operacionais.

Entretanto, em que pese à reforma implantada, a Lei n..$^{\circ}$ 8.630/93 praticamente não abordou a questão tarifária dos portos, deixando a cargo das administrações dos portos organizados adotarem estruturas de tarifas adequadas aos sistemas operacionais dos portos, em substituição à estrutura prevista no Decreto n. ${ }^{\circ} 24.508 / 34$. Os novos modelos deveriam ser submetidos à apreciação dos respectivos CAP para aprovação. Verificou-se que a estrutura tarifária aplicada sobre as operações desenvolvidas na área do porto organizado manteve diversas tabelas agregadas em dois grandes grupos: (a) utilização da infraestrutura (marítima, de acostagem e terrestre); e (b) prestação de serviços e de facilidades (movimentação de mercadorias, armazenagem, equipamentos portuários e diversos).

O Decreto n. ${ }^{\circ}$ 6.620/2008 reforçou a importância sobre essa temática, ao estabelecer que a exploração do porto organizado seja remunerada por meio de tarifas portuárias, que devem ser isonômicas para todos os usuários de um mesmo segmento, bem como por receitas patrimoniais ou decorrentes de atividades acessórias ou complementares. Ainda, as tarifas praticadas, inclusive dos serviços de natureza operacional e dos serviços denominados acessórios, deverão ser de conhecimento público e de fácil acesso. Inclusive, no rol das diretrizes gerais aplicáveis ao setor portuário marítimo consta a que se refere à promoção da sustentabilidade econômico-financeira da atividade portuária e implantação de sistema de preços e tarifas com base em centros de custos e eficiência operacional.

Observa-se, porém, que nada foi dito a respeito da formação da tarifa portuária, em que pese alguns portos relacionar quais os custos inseridos em cada uma das tabelas, sem, contudo, informar os respectivos valores. Ou seja, as tarifas portuárias são ainda hoje construídas de forma ad hoc. Ademais, as atuais tarifas portuárias, agrupadas em tabelas tarifárias, recebem denominações diferentes entre os diferentes portos brasileiros. De certo modo as oscilações tarifárias são decorrentes de estratégias de competição entre portos e não de uma proposta 
metodológica transparente de precificação com base nos custos portuários. Da Silva e Rocha (2012) estudaram esta competição para o caso entre os portos do Sul e Sudeste utilizando séries temporais de demanda por inexistência de uma forma de determinar o preço praticado pelos portos, levando a concluir que há em certa medida competição entre portos do sul do Brasil.

Sem um modelo teórico de tarifação cede-se lugar, inclusive, para abusos econômicos, tais como exercício de poder de mercado ou formação de conluios. Isso dificulta o papel das autoridades, especialmente, da Agência Nacional de Transportes Aquaviários (ANTAQ), criada pela Lei n. ${ }^{\circ} 10.233 / 01$, para atuar sobre os portos organizados, tanto na promoção de estudos aplicados às definições de tarifas, preços e fretes, em confronto com os custos e os benefícios econômicos transferidos aos usuários pelos investimentos realizados como para a definição de regulamentos a serem seguidos pelos componentes interno e externo ao porto.

Este artigo propõe um modelo teórico padrão de formação de tarifas portuárias. Uma vez que os portos não têm mais custos variáveis, não se pode usar a teoria microeconômica tradicional para determinar a tarifa portuária, ou seja, ganhos marginais não são observados. No lugar, a tarifa será formada a partir do princípio dos custos médios. O modelo proposto tem fundamentos de contabilidade de custos (Maher, 2001; Martins, 2003) e de contabilidade gerencial (Atkinson et al, 2000; Iudícibus, 1998) e de finanças corporativas (Brigham e Houston, 1999; Ross et al, 2007). O modelo proposto é compatível com a obtenção de lucros, visando conferir aos portos brasileiros auto-sustentação financeira. Saliente que Meersman, Van de Voorde e Vanelslander (2002) apresentam uma revisão pormenorizada dos modelos de tarifa portuária, mas nenhum deles usa os conceitos que fundamentam o modelo ora proposto.

O artigo está divido em quatro seções, além desta seção introdutória. A Seção 1 discute os conceitos de contabilidade de custos, contabilidade gerencial e finanças corporativas que serão utilizados pelo modelo. A Seção 2 apresenta o modelo. Na Seção 3 são feitas algumas considerações sobre a aplicabilidade do modelo, bem como sobre suas vantagens e desvantagens. A seção conclusiva encerra o artigo. 


\section{Fundamentação Teórica: Conceitos de Contabilidade de Custos e Gerencial e de Finanças Corporativas}

Os conceitos de contabilidade de custos que serão usados na construção do modelo são: departamentalização; objeto de custo; departamento de serviço e usuário; custos diretos; custos indiretos; rateio; base de rateio; e direcionadores de custos (Maher, 2001; Martins, 2003).

Os departamentos são objetos de custos, itens aos quais custos são atribuídos. Outros exemplos de objetos de custos são: um produto; e uma linha de produtos. Os departamentos apresentam custos diretos e indiretos:

i. Qualquer custo que pode ser relacionado diretamente com um objeto de custo é um custo direto.

ii. Qualquer custo que não pode ser relacionado diretamente com um objeto de custo é um custo indireto.

Os departamentos são divididos em: departamento de serviço; e departamento usuário. Por definição, os departamentos de serviços são aqueles que fornecem serviços a outras unidades na organização e os usuários utilizam os serviços dos departamentos de serviços.

Os custos dos departamentos de serviços são distribuídos aos departamentos usuários por meio de rateio. $\mathrm{O}$ rateio de custo representa a atribuição de um custo do departamento de serviço a um departamento usuário, segundo certa base. Uma base de rateio é uma medida relacionada com dois ou mais departamentos usuários, utilizada para ratear custos dos departamentos de serviços a esses objetos.

Então, supõe-se que os portos sejam departamentalizados em quatro departamentos usuários e um de serviço, conforme Tabela 1. 
Tabela 1 - Departamentalização dos Portos

\begin{tabular}{l|l}
\hline Departamento Usuário & Departamento de Serviço \\
\cline { 1 - 1 } 1. Acesso aquaviário & \multirow{2}{*}{ 5. Administração portuária } \\
\cline { 1 - 1 } 2. Atracação (acostagem) & \\
\cline { 1 - 1 } 3. Acesso terrestre & \\
\hline 4. Serviços diversos & \\
\hline
\end{tabular}

Note-se que o departamento que efetivamente existe nos portos é o departamento de número 5, Administração Portuária, os demais são hipotéticos, sendo relacionados na contabilidade gerencial.

Os custos do departamento Administração Portuária são rateados entre os quatro departamentos usuários (na Seção 2, definem-se os custos da Administração Portuária). A escolha da base de rateio é a fase mais difícil do rateio de custos, de acordo com a qual os custos são rateados aos departamentos usuários (Maher, 2001).

Usualmente, os custos dos departamentos de serviços são rateados com base em direcionadores de custos, ou seja, fatores que causam custos, por exemplo: mão de obra; máquinas; quantidade de faturas emitidas; quantidade de navios atendidos etc.

Vale lembrar que os quatro departamentos usuários são hipotéticos e, claro está, que não apresentam direcionadores de custos explícitos. Mas, com o auxílio da contabilidade gerencial (Atkinson et al, 2000; Iudícibus, 1998), pode-se calcular custos totais de operação e manutenção $(C O \& M)$ para cada um deles.

A Tabela 2 sugere uma lista não exaustiva de itens de custos portuários de operação e manutenção $(C O \& M)$ segundo o departamento usuário do porto. 
Tabela 2 - Itens de Custos Portuários de Operação e Manutenção por Departamento Usuário ${ }^{2}$

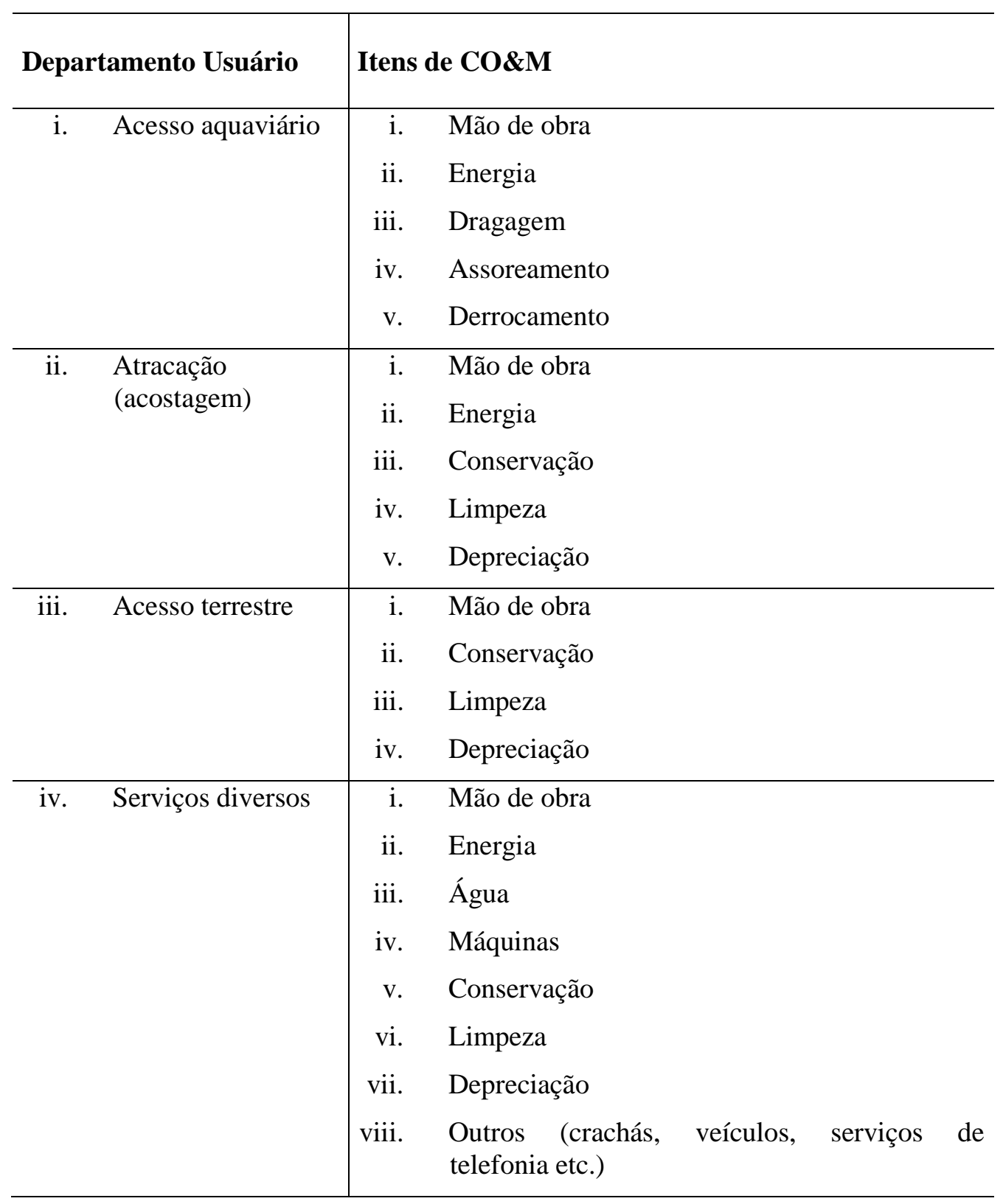

Portanto, é adotado como base de rateio a variável $C O \& M$. Isto é, o rateio será dado pela equação (1):

\footnotetext{
${ }^{2}$ Fonte: autores.
} 


$$
\lambda_{\mathrm{i}}=\frac{\mathrm{CO \& M}_{\mathrm{i}}}{\sum_{1}^{4}{\mathrm{CO} \& \mathrm{M}_{\mathrm{i}}}^{4}}
$$

onde $\lambda_{i}$ é o fator de rateio do departamento usuário $i$ e $C O \& M_{i}$ é o custo total de operação e manutenção do departamento usuário $i$.

Os principais conceitos de finanças empregados neste artigo são: valor presente, perpetuidade crescente, modelo de precificação de ativos (CAPM) e custo médio ponderado de capital (WACC).

\section{O Modelo}

Dada a inexistência de custos variáveis nos portos, não se pode recorrer à teoria microeconômica marginalista para modelar a tarifa portuária. Alternativamente, recorre-se ao princípio dos custos médios (Koutsoyiannis, 1992). Então, a tarifa portuária é dada pela equação (2):

$$
T P D_{i}=\bar{C}_{i} \times(1+\delta), \mathrm{i}=1,2,3,4
$$

onde $T P D_{i}$ é a tarifa portuária do departamento usuário $i, \bar{C}_{i}$ é o custo médio do departamento usuário $i$ e $\delta$ é o retorno exigido do porto ou custo de oportunidade portuário. Haralambides (2002) e Jansson e Rydén (1979) defendem que os preços portuários incluam o custo de oportunidade do porto.

O custo médio do departamento usuário $i$ é definido, consoante a equação (3):

$$
\bar{C}_{i}=\frac{C O \& M_{i}+\lambda_{i} C_{5}}{\theta_{i}}, \mathrm{i}=1,2,3,4
$$

onde $C_{5}$ é o custo do departamento de serviço (Administração Portuária) e os $\theta_{i}$, associados aos departamentos usuários, são dados conforme sugerido na Tabela 3 (as demais variáveis foram definidas anteriormente): 
Tabela 3 - Especificação dos $\theta_{i}$ associados aos departamentos usuários $^{3}$

\begin{tabular}{cll}
\hline DepartamentoUsuário & $\theta_{\mathrm{i}}$ \\
\hline i. $\quad$ Acesso aquaviário & Quantidade de navios $x \mathrm{TPB}^{1}$ (médio) \\
\hline ii. $\quad$ Atracação (acostagem) & Quantidade de navios $x$ tempo médio de atracação \\
\hline iii. $\quad$ Acesso terrestre & Quantidade de trens (caminhões) $x$ tempo médio no porto \\
\hline iv. $\quad$ Serviços diversos & Máquinas $x$ tempo médio de utilização \\
\hline
\end{tabular}

Os $\theta_{i}$ seriam obtidos de estatísticas portuárias históricas ou tomados de portos congêneres.

Agora, substituindo a equação (3) em (2), obtém a tarifa portuária do departamento usuário, de acordo com a equação (4).

$$
T P D_{i}=\left(\frac{C O \& M_{i}+\lambda_{i} C_{5}}{\theta_{i}}\right) \times(1+\delta), \mathrm{i}=1,2,3,4
$$

Enfim, os portos brasileiros teriam tantas tarifas quantos departamentos usuários, conforme a equação (4).

\subsection{Custos do departamento de serviço e retorno exigido do porto}

Define-se o custo do departamento de serviço (Administração Portuária), $C_{5}$, como o valor presente de uma perpetuidade crescente (Brigham e Houston, 1999; Ross et al, 2007), como representado na Equação 5:

$$
C_{5}=\frac{c_{5}}{(1+\delta)}+\frac{c_{5}(1+g)}{(1+\delta)^{2}}+\frac{c_{5}(1+g)^{2}}{(1+\delta)^{3}}+\ldots+\frac{c_{5}(1+g)^{n-1}}{(1+\delta)^{n}}+\ldots
$$

Em que $c_{5}$ (custos não-fabris) engloba os custos administrativos para gerir o porto e suprir o apoio administrativo, incluindo os custos: com os salários dos diretores e dos auxiliares administrativos; dos serviços jurídicos; do departamento comercial; dos serviços financeiros, contábeis e de processamento de dados; de ocupação desses setores; e dos investimentos

\footnotetext{
${ }^{3} \mathrm{TPB}=$ tonelagem de porte bruto. Fonte: autores.
} 
necessários e g é a taxa de crescimento anual de custos não-fabris; $c_{5}$ pode ser obtido facilmente das demonstrações contábeis históricas do porto.

Como a equação (5) é uma perpetuidade ${ }^{4}$, então, ela pode ser escrita na forma da expressão (6) considerando que $\delta>\mathrm{g}$ :

$$
C_{5}=\frac{c_{5}}{\delta-g}
$$

Importa registrar que os custos do departamento de serviço $C_{5}$ devem ser distribuídos entre tarifas e aluguéis de áreas e instalações portuárias.

Posto isso, a variável que entra no cálculo da tarifa portuária, dada pela equação (4), está expressa pela equação (7):

$$
C_{5}^{T}=\alpha_{T} C_{5}
$$

Onde $C_{5}^{T}$ é o custo do departamento de serviço atribuído a tarifa e $\alpha_{T}$ é uma fração $\left(\alpha_{T}=1\right.$ $\alpha_{A}$ ); sendo que $\alpha_{A}$ é a parcela de $C_{5}$ que vai para os arrendamentos. Considere-se que $\alpha_{j}(j=T, A)$ seja uma variável de política do porto.

A literatura especializada oferece diversas formas para calcular o retorno exigido de empresas quer de capital aberto, quer não-negociada ou de capital fechado (Copeland, Koller e Murrin, 2002; Damodaran, 2008).

Para calcular $\delta$ de uma empresa de capital aberto usualmente empregam-se os modelos CAPM (Capital Asset Pricing Model) e WACC (Weighted Average Cost of Capital). Já para o $\delta$ de uma empresa não-negociada usa-se, juntamente com o WACC, uma das seguintes situações: (a) betas de empresas comparáveis; (b) betas contábeis; e (c) betas a partir de fundamentos financeiros (Damodaran, 2008).

Alternativamente, dado que a atividade portuária pública envolve pouco, ou nenhum, risco financeiro para o capital social alocado, $\delta$ poderia ser igual à média das taxas internas de retorno dos títulos governamentais de longo prazo, ponderadas pelo prazo de vencimento

\footnotetext{
${ }^{4}$ a expressão (5) é ,matematicamente, uma série geométrica infinita, portanto passível de ser expressa tal como (6).
} 
desses títulos, posto que os retornos pagos pelos títulos do Tesouro são, em tese, livres de risco.

\section{Algumas Considerações Sobre o Modelo}

O modelo tarifário proposto cobre os custos totais do porto, além de oferecer uma margem de lucro (retorno exigido). Portanto, o modelo é compatível com a sustentação econômicofinanceira dos portos. Entretanto, o custo de oportunidade dos portos é de difícil mensuração uma vez que as decisões de investimento não são baseadas apenas em taxas de juros, mas também de expectativas de crescimento da movimentação portuária que pode ou não ocorrer. Desta feita, sugere-se um pormenorizado estudo sobre como quantificá-lo.

Os portos brasileiros passariam ter as mesmas denominações de tarifas portuárias e, o que é melhor, seria conhecido como elas são calculadas. Todos os portos teriam tantas tarifas quantos departamentos usuários identificados. O modelo padroniza a forma de calcular a tarifa portuária.

Com o modelo, podem-se recalcular as tarifas sempre que houver alterações significativas nos custos de operação e manutenção e nos $\theta_{i}$ (ver equação 3 ), sem falar da calibragem do retorno exigido.

O modelo impõe aos portos uma melhoria na gestão dos custos e das receitas. Portos ineficientes, do ponto de vista de custos, teriam tarifas relativamente elevadas. O que poderia provocar reduções no seu market-share. Isso poderia acontecer também se ocorressem abusos na tarifação objetivando ganhos extraordinários.

Diga-se que para o modelo funcionar plenamente o porto precisaria possuir sistemas de custeamento e de contabilidade gerencial integrados com os sistemas de estatística portuária. Talvez, a falta de sistemas de custeio e de estatística nos portos seja a principal fraqueza operacional do modelo. Sem eles, o modelo não pode ser posto em prática.

Vale lembrar que os sistemas de custeamento são elementos cruciais para o alcance da competitividade empresarial, dizem os especialistas (Atkinson et al, 2000; Iudícibus, 1998). 


\section{Conclusão}

Este artigo apresenta um modelo teórico padrão de formação de tarifas portuárias. A tarifa é baseada nos princípios dos custos médios, da contabilidade de custos, da contabilidade gerencial e de finanças corporativas. Foram adotados tais princípios para a formação da tarifa porque com a lei de modernização dos portos, os custos variáveis portuários deixaram de existir. Daí, não se pode aplicar a teoria microeconômica tradicional, a teoria marginalista, para calcular as tarifas portuárias. Outra modelagem também utilizada em infraestrutura é a regra do custo total, portanto passível de uma proposta alternativa de modelo de tarifa para portos.

Pode-se concluir que o modelo é de fácil entendimento e aplicação, desde que os portos possuam sistemas integrados: de custeamento, de contabilidade gerencial e de estatística portuária.

Com o modelo os custos totais do porto são cobertos, inclusive os custos de investimentos. O modelo é compatível com a obtenção de lucros.

Por último, é preciso dizer que o modelo não trata da cobrança junto aos operadores portuários pela movimentação de cargas e nem da questão de arrendamentos portuários. Esses temas merecem uma discussão separada. Rocha (2005) e Rocha, Gartner e Cavalcante (2011), por exemplo, oferecem modelos de precificação de aluguéis de áreas e instalações portuárias no caso do Brasil. 


\section{Referências}

Aragão, A. (2004) Agências reguladoras e a evolução do direito administrativo e econômico. $4^{\mathrm{a}}$ ed., Rio de Janeiro: Forense.

Atkinson, A. A., Banker, R. D., Kaplan, R. S. e Young, S. M. (2000) Contabilidade gerencial. São Paulo: Atlas.

Castro, N. (2000) Privatização do setor de transportes no Brasil. In: Pinheiro, A. C. e Fukasaku, K. A privatização no Brasil: o caso dos serviços de utilidade pública. Rio de Janeiro: BNDES.

Copeland, T., Koller, T., e Murrin, J. (2002) Avaliação de empresas. São Paulo: Makron Books.

Da Silva, F. G. F. e Rocha, C. H. (2012) A demand impact study in Southern and Southeastern ports in Brazil: an indication of port competitiveness. Maritime Economics \& Logistics, vol. 14, pp. 204-219.

Damodaran, A. (2008) Avaliação de investimentos: ferramentas e técnicas para a determinação do valor de qualquer ativo. Qualitymark: Rio de Janeiro.

Haralambides, H. E. (2002) Competition, excess capacity, and the pricing of port infrastructure. International Journal of Maritime Economics, vol. 4, pp. 323-347.

Iudícibus, S. (1998) Contabilidade Gerencial. São Paulo: Atlas.

Jansson, J. O. e Rydén, I. (1979) Swedish seaports: economics and policy. The Economic Research Institute, Stocokholm School of Economics, Stockholm.

Koutsoyiannis, A. (1992) Modern microeconomics. Londres: MacMillan.

Maher, M. (2001) Contabilidade de custos: criando valor para a administração. São Paulo: Atlas.

Martins, E. (2003) Contabilidade de custos. São Paulo: Atlas.

Meersman, H., Van de Voorde, E. e Vanelslander, T. (2002) Port pricing issues: considerations on economic principles, competition and wishful thinking. Bruxelas: Imprint-Europe.

Pêgo Filho, B., Cândido Júnior, J. O., Pereira, F. (1999) Investimento e financiamento da infraestrutura no Brasil: 1990/2002. Texto para discussão n. 680. Brasília: IPEA.

Rocha, C. H. (2005) Uma proposta de precificação de arrendamentos de áreas portuárias. Anais do $5^{\circ}$ Congresso USP de Controladoria e Contabilidade, São Paulo.

Ross, S. A., Westerfield, R. W. e Jeffrey F. J. (2007) Administração financeira: corporate finance. São Paulo: Atlas.

Rossetti, J. P., Contador, C. R., Villaça, M. J. e Soudine, R. M. (1985) Economia de mercado: fundamentos, falácias e valores. Rio de Janeiro: IBMEC/CODIMEC.

Rocha, C. H., Gartner, I. R. e Cavalcante, L. R. (2011) A model of lease of port areas: a new contribution. Journal of Transport Literature, vol. 5, n. 3, pp. 4-15. 